\title{
Silicon-standard-based Comparisons of LEAP Accuracy and Precision
}

Ty Prosa

\section{CAMECA Instruments Inc., Madison, Wisconsin, United States}

The Pre-Sharpened Microtip ${ }^{\mathrm{TM}}$ (PSM) [1,2] is the closest thing the atom-probe community has to a reference standard. Its chemical purity, exact atomic structure/orientation, and high survivability under voltage-pulsing and laser-pulsing conditions makes it ideal for use by the manufacturer (CAMECA ${ }^{\circledR}$ ) for acquisition software development, LEAP ${ }^{\circledR}$ factory acceptance testing, and hardware assessment by service in the field. However, the potential for this sample to serve as an analytical standard for data quality, composition, accuracy and precision methods development, etc., has been relatively unexplored.

This study investigates the suitability for the commercially available PSM to serve as an analytical reference standard for atom probe tomography (APT). LEAP data were collected on multiple instruments, platforms, and under various analysis conditions. Automated data reduction methods were developed to enable a systematic understanding of best practices to achieve highly comparable results of various material characteristics including silicon isotope fractions, dopant levels, background characteristics, and multi-hit performance, all of which will be discussed in this presentation.

Figure 1 shows the results of ${ }^{29} \mathrm{Si}$ isotopic-fraction measurements from three different LEAP 5000XR and three LEAP 5000XS instruments (with some repeats). In order to achieve comparable results, consistent (automated) ranging, careful (automated) background correction, and deadtime correction procedures all play an important role for achieving highly precise and accurate results. In this case significant differences in isotopic-fraction measurements are evident depending on application of a deadtime correction, similar to observations made in determining ${ }^{13} \mathrm{C} /{ }^{12} \mathrm{C}$ isotopic rations in meteoritic nanodiamonds [3]. Without deadtime correction, measurement-to-measurement differences for the straight-flight-path instrument are observed to significantly exceed estimated precision, while the reflectron measurement have much less variability. However, statistically consistent and precise quantification is achievable for all data when deadtime considerations are considered. Here, the final measurement precision is comparable to the scatter predicted by simple counting statistics and error propagation. 


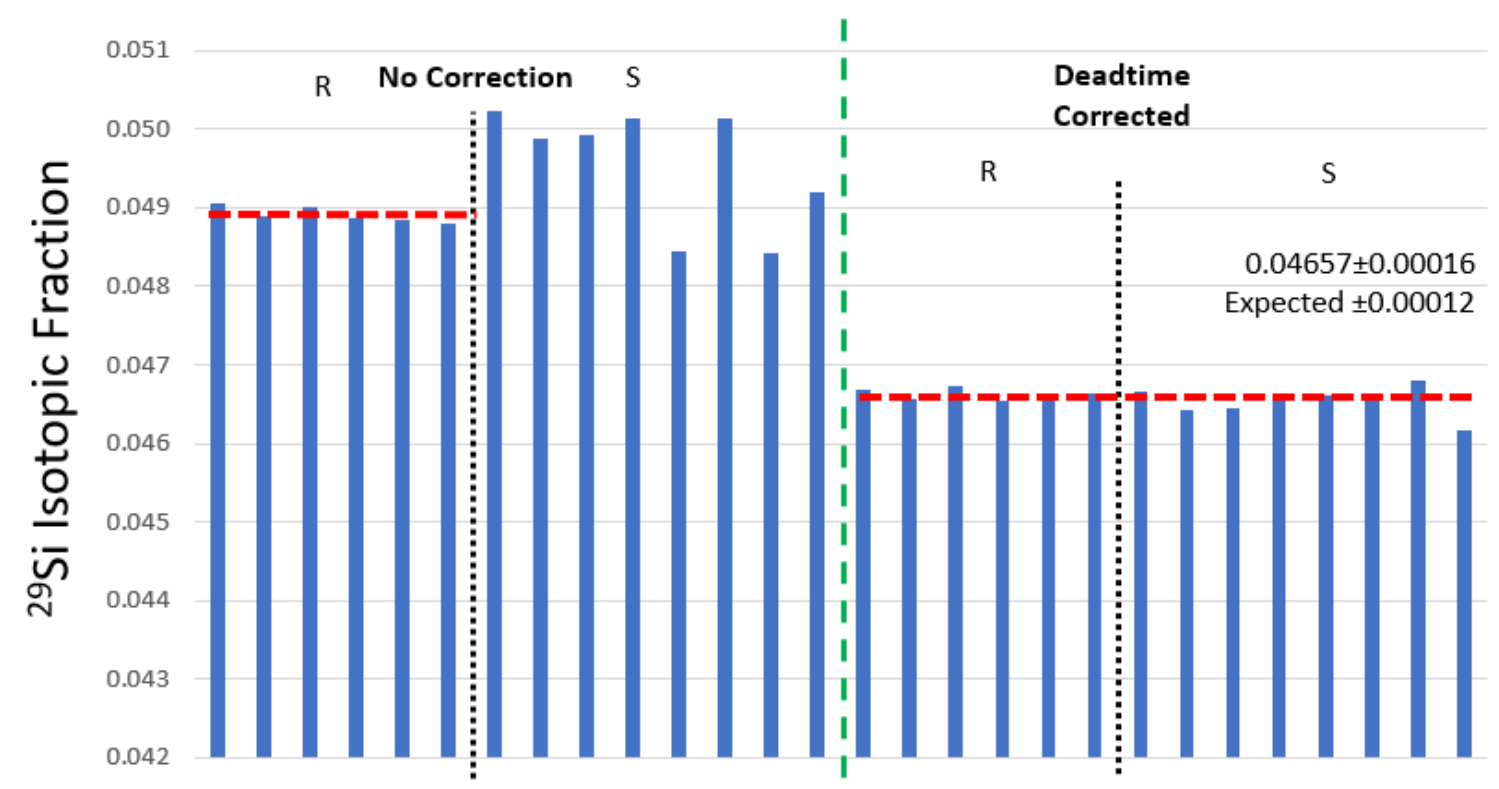

Figure 1. Measurement of the ${ }^{29} \mathrm{Si}$ isotopic fraction as measured from three LEAP 5000XR and three LEAP 5000XS instruments with some repeats. By utilizing appropriate and consistent data reduction methods, high precision results can be achieved. In this figure the need for appropriate deadtime correction is illustrated. Without deadtime correction (left) the reflectron data is very consistent and has high precision while the straight flight-path data has significant scatter. After deadtime correction, all of the separate measurements agree to nearly the precision predicted by simple propagation of errors and counting statistics $(0.4567 \pm 0.0016$ actual and \pm 0.0012 expected $)$.

\section{References}

[1] K. Thompson, D. J. Larson and R. M. Ulfig, Microscopy and Microanalysis 11(S2) (2005) p. 882.

[2] R. Shivaraman et al., Microscopy and Microanalysis 15(S2) (2009) p. 296.

[3] J.B. Lewis et al., Ultramicroscopy 159 (2015) p. 248. 